\title{
Dynamics of the Dow Jones and the NASDAQ stock indexes
}

\author{
Fernando B. Duarte • J.A. Tenreiro Machado • \\ Gonçalo Monteiro Duarte
}

\begin{abstract}
The goal of this study is the analysis of the dynamical properties of financial data series from worldwide stock market indices. We analyze the Dow Jones Industrial Average $\left({ }^{\wedge} D J I\right)$ and the NASDAQ Composite $\left({ }^{\wedge} I X I C\right)$ indexes at a daily time horizon. The methods and algorithms that have been explored for description of physical phenomena become an effective background, and even inspiration, for very productive methods used in the analysis of economical data. We start by applying the classical concepts of signal analysis, Fourier transform, and methods of fractional calculus. In a second phase we adopt a pseudo phase plane approach.
\end{abstract}

Keywords Pseudo phase plane · Fourier transform . Power law $\cdot$ Fractional calculus

\section{Introduction}

The study of fractional order systems has received considerable attention, due to the fact that many physical systems are well characterized by fractional models. The importance of fractional-order mathematical model is that it can be used to make a more accurate description and it can even give a deeper insight into the processes underlying long-range memory behavior $[9,11,16]$. It seems that there are many distinct analogies between the dynamics of complex physical and economical or even social systems. The methods and algorithms that have been explored for description of physical phenomena become an effective background and inspiration for very productive methods used in the analysis of economical data $[8,14,17,18]$.

In this paper we study the Dow Jones and the NASDAQ indexes at a daily time horizon at the closing.

People on Wall Street found it difficult to analyze the daily jumble of up-a-quarter and down-aneighth, or whether stocks generally were rising, falling or staying even. Charles Dow a journalist, neither financier nor broker, devised his stock average to make sense of this confusion. He began in 1884 with eleven stocks, most of them railroads. Railroads were among the biggest and sturdiest companies in America at that time, which is why they dominated Dow's first average. Few stocks of industrial companies were publicly traded, and those were considered highly speculative. On May 26, 1896, he introduced the industrial average. In October of that year, Dow's original average 
shed the last of its non-railroad stocks and became the twenty-stock railroad average. To complete this line of history, the utilities average came along in 1929 and the railroad average was renamed the transportation average in 1970. Nowadays, of course, there are plenty of indicators to tell investors what the stock market is doing. The Dow Jones Industrial Average is in sync with other major market barometers. That is true despite the difference in computation methods; the Dow is unweighted while almost all other indexes weight their stocks by market capitalization, which is price times shares outstanding. It is also true despite the fewer number of stocks in the Dow [1].

The NASDAQ (National Association of Securities Dealers Automated Quotation) Stock Market, founded in 1971, was the world's first electronic stock market. The purpose of its founding was to popularize the OTC (over-the-counter) securities market which, up to that point, had been relatively unknown and unused by many stock players. With its first day of trading on February 8, 1971, the NASDAQ system displayed quotes for over 2500 over-the-counter stocks. The NASDAQ stock market is full of technology stocks of up-and-coming companies, some with expensive stock prices and some for just pennies. It continues to be America's most popular market in a day and age when technology still seems to be the wave of the future [2].

Bearing these ideas in mind, this paper is organized as follows: Sects. 2 and 3 respectively present some fundamental concepts, and the dynamical analysis. The existence of a power law relationship typical of systems with fractional calculus is shown. Finally, Section 4 draws the main conclusions and addresses perspectives towards future developments.

\section{Fundamental concepts}

In this section we present a review of fundamental concepts involved in the experiments. The technique used to determine the fractional behavior of the Dow Jones [3] and NASDAQ [4] index signals is based on the slope of their trendlines in the frequency spectrum. Additionally, the pseudo phase space $(P P S)$ is obtained using the method of the time delays $[7,10]$.

\subsection{Fourier transforms}

In order to examine the behavior of the signal spectrum, a power law trendline is superimposed upon the
Fourier Transform (FT) signal. To study the signal spectrum, we approximate the modulus of the FT amplitude through power functions

$|\mathcal{F}\{x(t)\}| \approx p \omega^{q}, \quad\{p, q\} \in \mathbb{R}$

where $F$ is the Fourier operator, $x(t)$ is the index and $t$ is time, $\omega$ the frequency, $p$ a positive constant that depends on the signal amplitude and $q$ is the trendline slope [12]. According to the values of $q$, the signals can exhibit an integer or fractional order behavior.

The standard Fourier transform describing the data in the 'Fourier domain' is precise in frequency, but not in time. Small changes in the signal at one location cause change in the Fourier domain globally. It is of interest to have transformed domains that are simultaneously precise, both in time and frequency domains.

The Windowed Fourier transforms (WFT) are important in providing simultaneous insight in time and frequency behavior of the signal. A window function is a function that is zero-valued outside of some chosen interval. When a signal is multiplied by a window function, the product is also zero-valued outside the interval: all that is left is the "view" through the window. In this paper, among the several window functions, we adopt the Gaussian window for a sliding-window Fourier transform. The coefficients of a Gaussian window are computed from the following equation:

$W(t)=e^{-\frac{1}{2}\left(\alpha \frac{t}{T / 2}\right)^{2}}$

where $T$ is the window length, $-\frac{T}{2} \leq t \leq \frac{T}{2}$, and $\alpha$ is the reciprocal of the standard deviation. The width of the window is inversely related to the value of $\alpha$ : a larger value of $\alpha$ produces a narrower window.

\subsection{Pseudo phase plane}

An essential problem in nonlinear time series analysis is to determine whether or not a given time series is a deterministic signal from a low-dimensional dynamical system. If it is, then further questions of interest are: what is the dimension of the phase space supporting the data set? Is the data set chaotic? The key to answering these questions is embodied in the method of phase space reconstruction, which has been rigorously justified by the embedding theorems. Takens' embedding theorem $[5,6]$ asserts that if a time series is one component of an attractor that can be represented by a smooth $d$-dimensional manifold (with $d$ an integer) 
then the topological properties of the signal are equivalent to the topological properties of the embedding formed by the $m$-dimensional phase space vectors

$y(t)=[s(t), s(t+\tau), s(t+2 \tau), \ldots, s(t+(m-1) \tau)]$

whenever $m>2 d+1$. In (3), $\tau$ is called the delay time and $d$ is the embedding dimension. Different choices of $t$ and $m$ yield different reconstructed trajectories. The vector $y(t)$ can be plotted in a $d$-dimensional space forming a curve in the PPS. If $d=2$, we get a two-dimensional time delay space where the signal $\{s(t), s(t+\tau)\}$ is related to the model $\{s(t), \dot{s}(t)\}$. In this case the $P P S$ it is called pseudo phase plane $(P P P)$. We expect, with the $P P P$ of the signal, to draw conclusions about the system dynamics [10].

There have been various proposals for choosing an optimal delay time, $\tau$, for topological properties based on the behavior of the autocorrelation function. These include the earliest time $t$ at which the autocorrelation drops to a fraction of its initial value or has a point of inflection. These definitions seek to find times where linear correlations between different points in the time series are negligible, but they do not rule out the possibility of more general correlations. Some argue that a better value for $\tau$ is the value that corresponds to the first local minimum of the mutual information. The mutual information is a measure of how much information can be predicted about one time series point giving full information about the other. The values of $\tau$ at which the mutual information has a local minimum are equivalent to the values of $\tau$ at which the logarithm of the correlation sum has a local minimum. It is not clear which method, if any, is superior for all topological properties. However, optimal values based on the behavior of the autocorrelation function is the easiest way to compute and is adopted in our experiments [13].

\section{Dynamics of financial indexes}

In this section we study numerically the signals corresponding to the Dow Jones and the NASDAQ indexes. For both signals we study the fractional behavior and the $P P P$ reconstruction.

Figures 1 and 2 depict the time evolution of the two indexes with the well-known noisy of chaotic-like characteristics.

\subsection{Fourier analysis}

Figure 3 shows the amplitude of the FT for the Dow Jones $\left(\left|\mathcal{F}\left\{x_{D}(t)\right\}\right|\right)$ signal index. A trendline is calculated and it is superimposed on the signal. For the Dow Jones index, the slope yields $q=-0.79$, revealing a fractional order behavior. Figure 4 shows the amplitude of the FT for the NASDAQ $\left|\mathcal{F}\left\{x_{N}(t)\right\}\right|$ signal index. A trendline is also calculated and superimposed on the signal. For the NASDAQ index, the slope yields $q=-0.83$ and reveals, also, a fractional order behavior. In fact, in both cases we get a fractional noise inbetween white and pink noise, corresponding to a considerable volatility.

We now adopt the windowed Fourier transform and we consider $\alpha=2.5, T=366$ days (one year) and
Fig. 1 The temporal evolution of the daily closing value of Dow Jones index, from October, 1928 to June, 2009

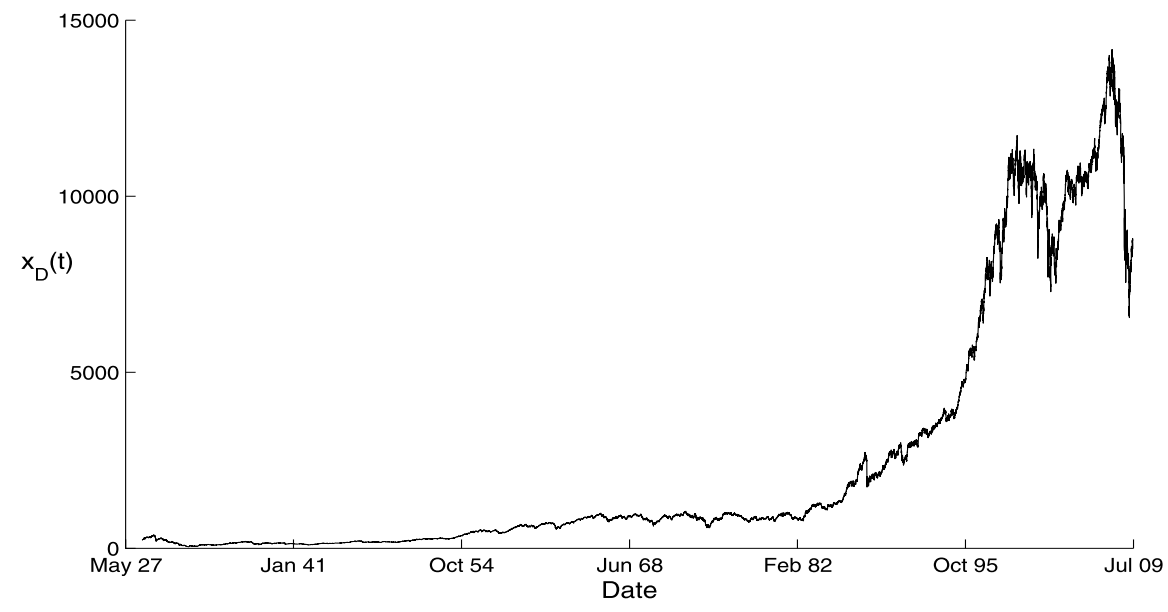


Fig. 2 The temporal evolution of the daily closing value of NASDAQ index, from February, 1971 to June, 2009

Fig. $3\left|\mathcal{F}\left\{x_{D}(t)\right\}\right|$ and the trendline for the Dow Jones signal index

Fig. $4\left|\mathcal{F}\left\{x_{N}(t)\right\}\right|$ and the trendline for the NASDAQ signal index
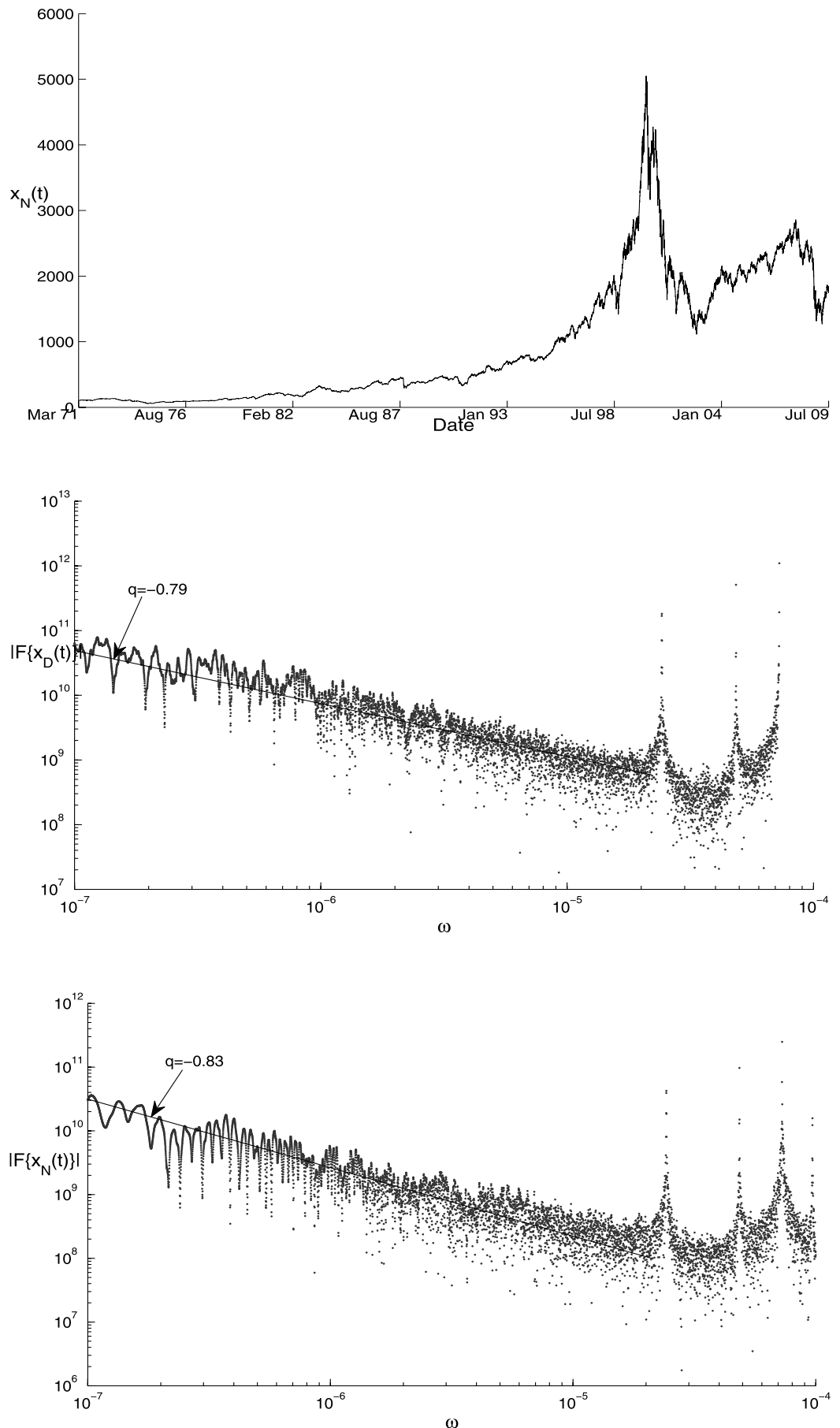

that two consecutive windows are superimposed over a range of window length of $\beta=50 \%$.

For the Dow Jones index we considerer a total of the 110 windows centered at $t=183 \lambda_{D}$ days for $\lambda_{D}=$ $1,2, \ldots, 110$ and for the NASDAQ index we adopt a total of 51 windows centered at $t=183 \lambda_{N}$ days for $\lambda_{N}=1,2, \ldots, 51$.

Figure 5 depicts the amplitude of a sliding-window Fourier transform, $\left|F_{w}\left\{x_{D}(t)\right\}\right|$, centered at $t=183 \lambda_{D}$ for $\lambda_{D}=\{10,20,30,40,50,60,70,80,90,100\}$, for 
Fig. 5 Windowed Fourier transform $\left|F_{w}\left\{x_{D}(t)\right\}\right|$ for Dow Jones index for $\alpha=2.5, T=366$ days and $\beta=50 \%$ centered at $t=183 \lambda_{D}$ for $\lambda_{D}=\{10,20,30,40,50,60$, $70,80,90,100\}$
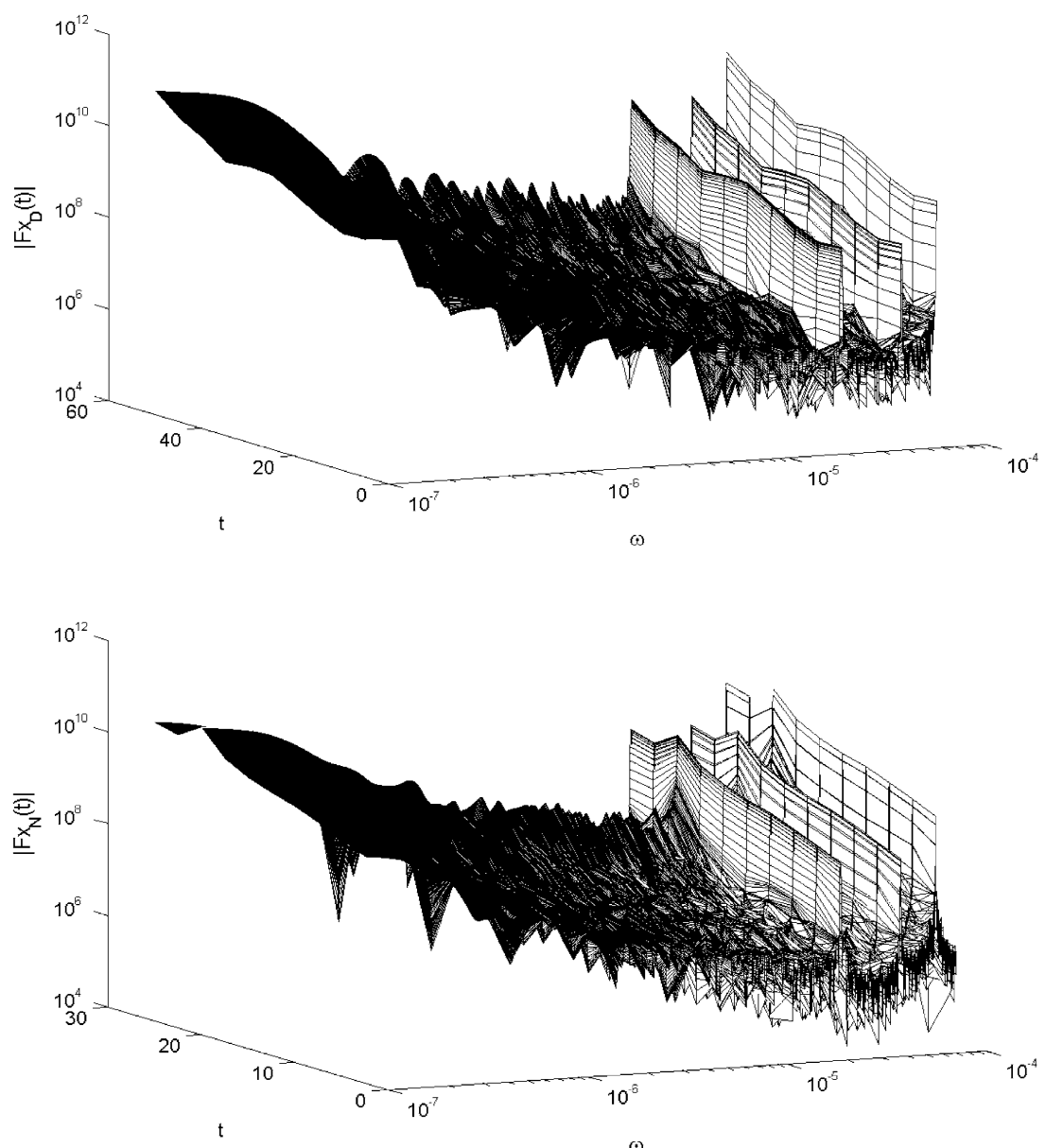

Fig. 6 Windowed Fourier transform $\left|F_{w}\left\{x_{N}(t)\right\}\right|$ for NASDAQ index for $\alpha=2.5, T=366$ days and $\beta=50 \%$, centered at $t=183 \lambda_{N}$ for $\lambda_{N}=\{5,10,15,20,25,30,35$, $40,45,50\}$ the Dow Jones index, while Fig. 6 shows the amplitude of a sliding-window Fourier transform, $\left|F_{w}\left\{x_{N}(t)\right\}\right|$, centered at $t=183 \lambda_{N}$ for $\lambda_{N}=\{5,10,15,20,25,30$, $35,40,45,50\}$.

For each of the above FT, a power trendline is calculated and superimposed on the signal. Table 1 shows the values of the slope $q$ for both indexes where a fractional order behavior is clearly observed.

We know that the lower the negative slope, the higher the attenuation of the high frequencies and, consequently, the smoother the time evolution of signal. Therefore, the Fourier transform analysis suggests that the NASDAQ index is more volatile than the Dow Jones index. This is in accordance with reality. In fact, the NASDAQ index tends to have a more variable quotation-it usually outpaces the Dow Jones index both to the upside and the downside. This is because the NASDAQ index is heavily weighted to technology stocks, containing more speculative (i.e., high
Table 1 Slope $q$ for the windowed Fourier transform, for the NASDAQ and the Dow Jones indexes

\begin{tabular}{ccccc}
\hline Dow Jones & & & \multicolumn{2}{c}{ NASDAQ } \\
\cline { 5 - 5 }$\lambda_{D}$ & $q$ & & $\lambda_{N}$ & $q$ \\
\hline 10 & -1.306 & 5 & -1.170 \\
20 & -1.374 & & 10 & -1.139 \\
30 & -1.339 & & 15 & -1.108 \\
40 & -1.374 & & 20 & -1.157 \\
50 & -1.444 & & 25 & -1.131 \\
60 & -1.263 & 30 & -1.138 \\
70 & -1.304 & 35 & -1.103 \\
80 & -1.128 & 40 & -0.968 \\
90 & -1.332 & 45 & -1.076 \\
100 & -1.269 & 50 & -1.029 \\
\hline
\end{tabular}

risk/high reward) companies than the Dow Jones index, which is constituted by large and stable compa- 
Fig. 7 Autocorrelation $\rho$ vs. time lag $\tau$, for the Dow Jones index
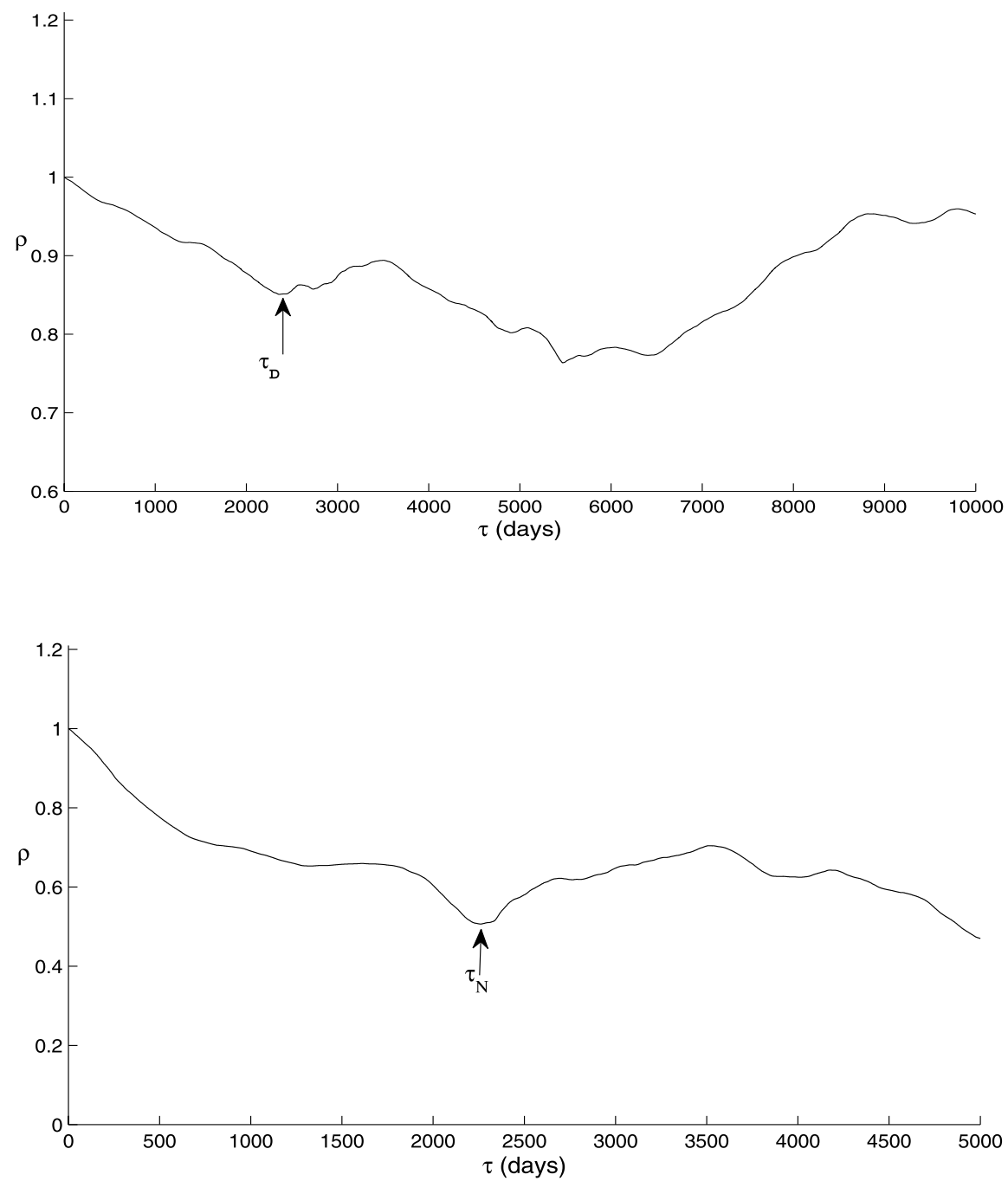

Fig. 8 Autocorrelation $\rho$ vs. time lag $\tau$, for the NASDAQ index nies. It is interesting to note, in the window Fourier transform, that the NASDAQ volatility has been increasing in the last years. However, this is not surprising if we have two aspects in mind: (i) volatility is normally seen in the market as a proxy for the investors' emotions and fears, and (ii) in the last decade we have witnessed two major crises and, therefore, investors have been quite anxious.

Peaks in the Fourier transforms' charts for both indexes are easily identifiable. They occur to the frequencies corresponding to 1 day $\left(\omega_{1}=7.27 E-\right.$ $5 \mathrm{rad} / \mathrm{sec}), 1.5$ days $\left(\omega_{2}=4.85 E-5 \mathrm{rad} / \mathrm{sec}\right)$ and 3 days $\left(\omega_{3}=2.42 E-5 \mathrm{rad} / \mathrm{sec}\right)$. This suggests the existence of a short-range periodicity in the stock market. In fact, $\omega_{3}$ corresponds simply to the sampling frequency and $\omega_{2}$, a cross-effect between $\omega_{1}$ and $\omega_{3}$. However, $\omega_{3}$ reveals that we have a half-week period limit cycle.

\subsection{Pseudo phase plane analysis}

In order to study the $P P P$ properties of the indexes $x_{D}(t)$ and $x_{N}(t)$, we adopt the earliest time $t$ at which the autocorrelation $\rho$ has a point of inflection. This value is the delay time, $\tau$, used for the $P P P$ construction [15].

Figures 7 and 8 depict the autocorrelation $\rho$ versus $\tau$, for the Dow Jones and NASDAQ indexes, respectively. For the Dow Jones index the first local mini- 
Fig. 9 Pseudo phase plane for Dow Jones index and $\tau_{D}=2330$

Fig. 10 Pseudo phase plane for NASDAQ index and $\tau_{N}=2174$
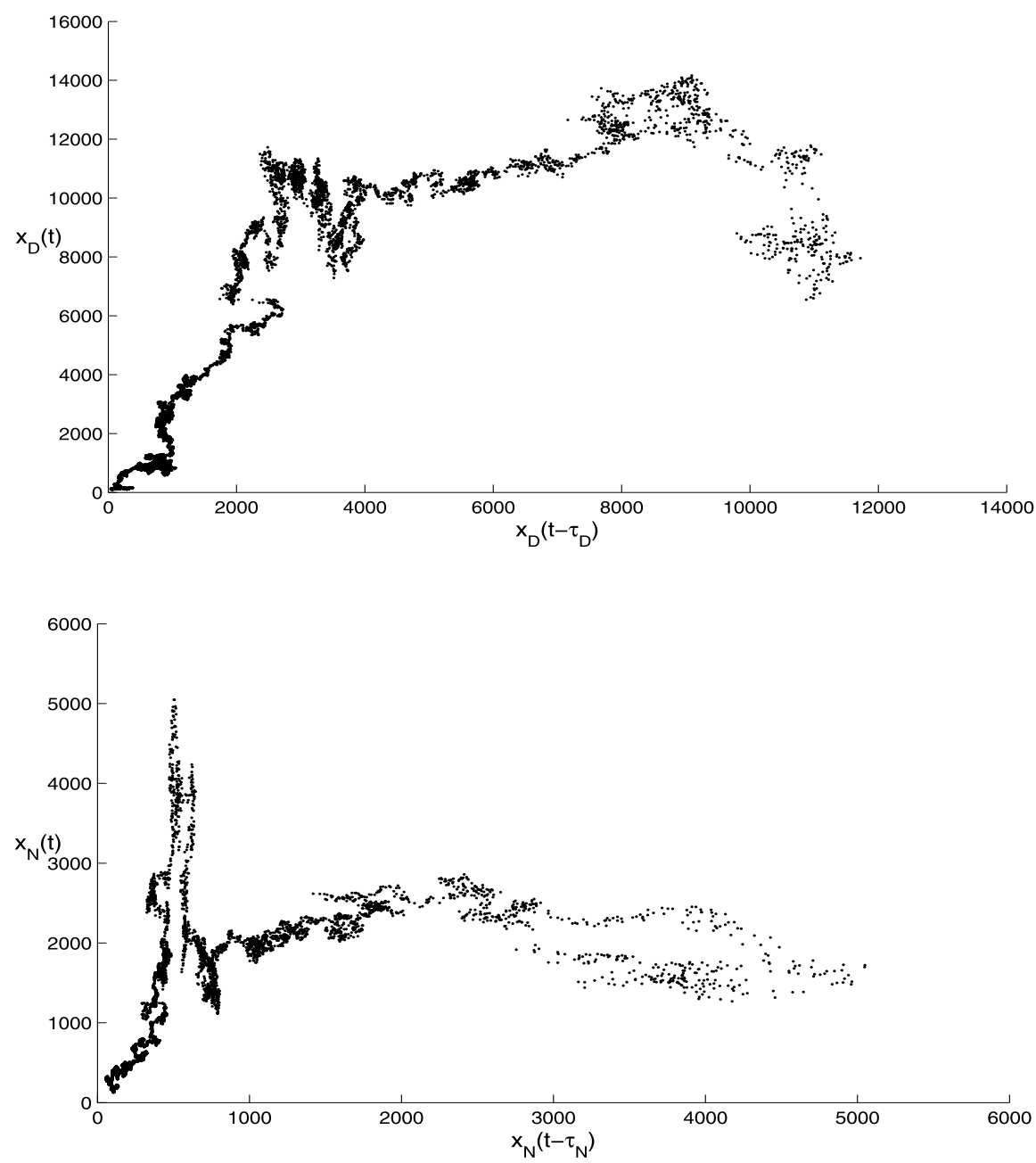

Table 2 Date of the Begin and the End time instants in each $P P P$ partition, for the NASDAQ and the Dow Jones indexes

\begin{tabular}{llllll}
\hline \multirow{2}{*}{ Partition } & \multicolumn{2}{l}{ Dow Jones } & & & NASDAQ \\
\cline { 2 - 3 } \cline { 5 - 6 } \cline { 5 - 6 } & Begin & End & & Begin & End \\
\hline 1 & $21 / 12 / 1932$ & $15 / 09 / 1976$ & $13 / 08 / 1971$ & $29 / 11 / 1990$ \\
2 & $20 / 09 / 1976$ & $10 / 09 / 1987$ & $30 / 11 / 1990$ & $02 / 08 / 1991$ \\
3 & $20 / 10 / 1987$ & $16 / 03 / 1994$ & $05 / 08 / 1991$ & $21 / 04 / 1995$ \\
4 & $17 / 03 / 1994$ & $13 / 05 / 1998$ & $24 / 04 / 1995$ & $05 / 04 / 1999$ \\
5 & $26 / 01 / 1988$ & $13 / 03 / 2000$ & $04 / 02 / 2000$ & $13 / 07 / 2000$ \\
6 & & & $22 / 04 / 1999$ & $03 / 02 / 2000$ \\
\hline
\end{tabular}

mum occurs for the time lag $\tau_{D}=2330$ days, while for the NASDAQ index it occurs for $\tau_{N}=2174$ days.

Figures 9 and 10 depict the $P P P$ values for the Dow Jones and NASDAQ indexes, for the chosen time lag.
Table 3 Parameters $\{a, b\}$ of the power law trendline for the $P P P$ partitions, for the NASDAQ and the Dow Jones indexes

\begin{tabular}{llllll}
\hline \multirow{2}{*}{ Partition } & \multicolumn{2}{l}{ Dow Jones } & & & NASDAQ \\
\cline { 2 - 3 } \cline { 6 - 6 } & $a$ & $b$ & & $a$ & $b$ \\
\hline 1 & 22.0338 & 0.5627 & 0.2823 & 1.415 \\
2 & 6.2494 & 0.8838 & 0.7073 & 1.384 \\
3 & 1308.2781 & 0.2482 & 8579377001 & -2.342 \\
4 & 691.1133 & 0.3202 & & 171.6524 & 0.3527 \\
5 & 3100.4451 & 0.1421 & 191.0417 & 0.2529 \\
6 & & & 6156.9266 & -0.1193 \\
\hline
\end{tabular}

The $P P P$ charts reveal that we can subdivide them into several different partitions. Based on a visual analysis of the pattern of the Dow Jones PPP chart we decided to consider five partitions as illustrated in Fig. 11. For 


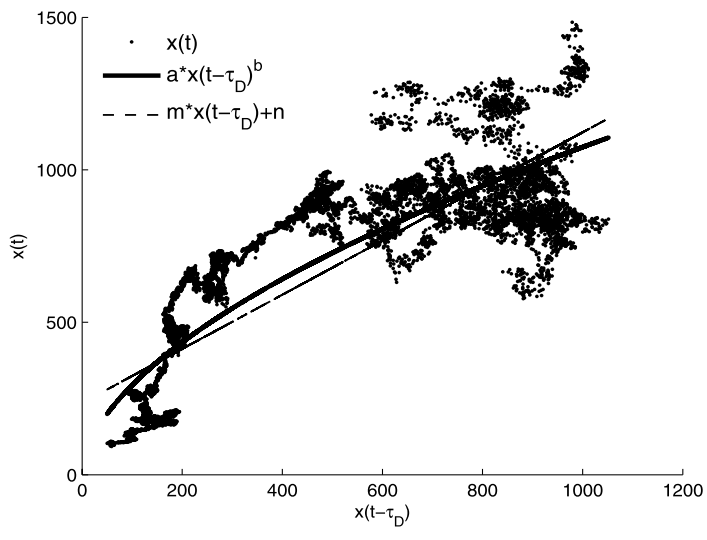

Partition 1

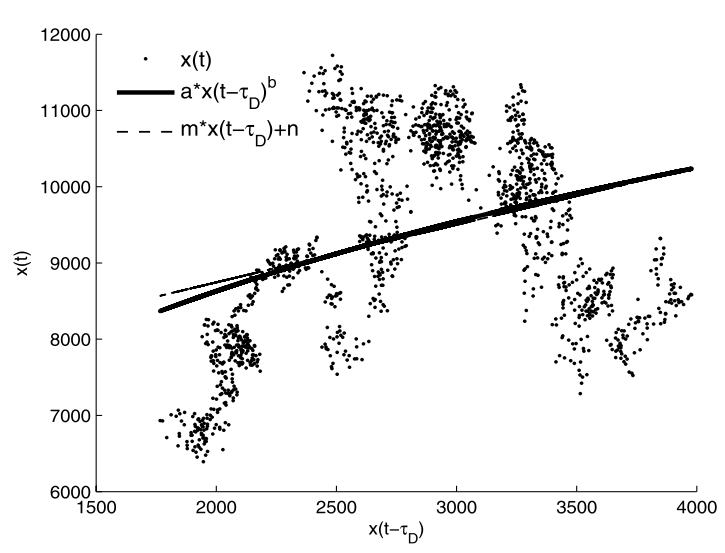

Partition 3

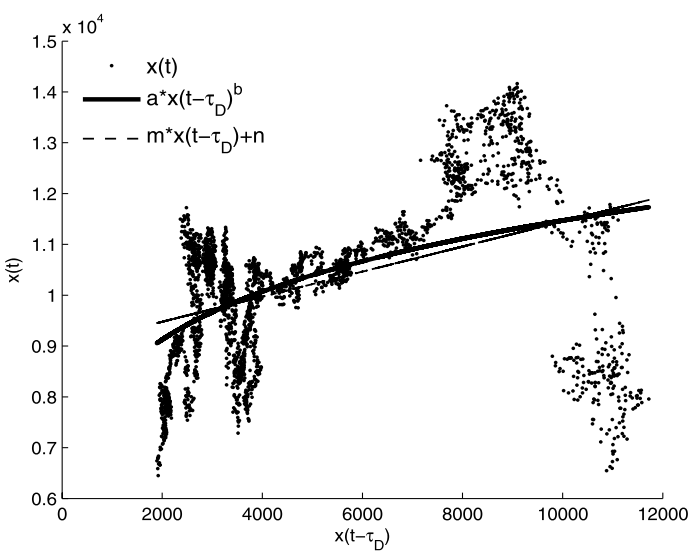

Partition 5

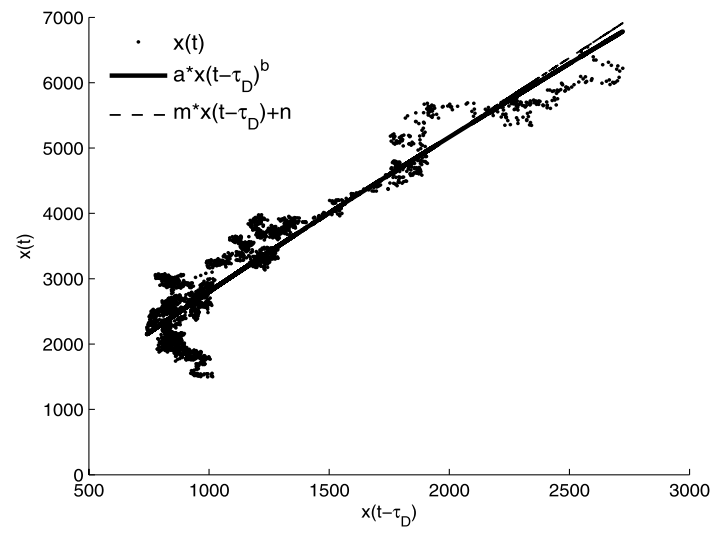

Partition 2

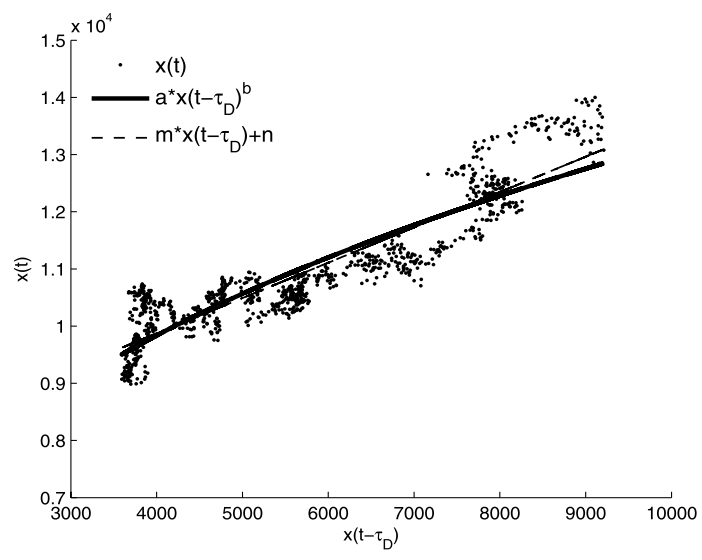

Partition 4

Fig. 11 The five partitions and corresponding power law and linear law trendlines for the Dow Jones index's $P P P$ 


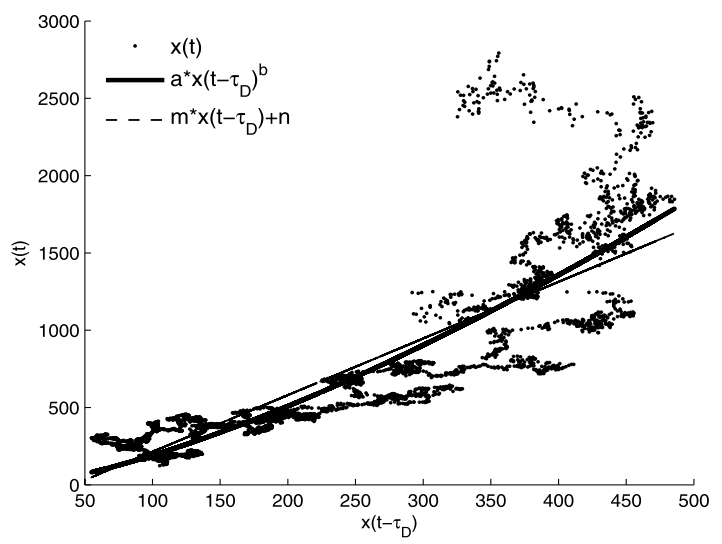

Partition 1

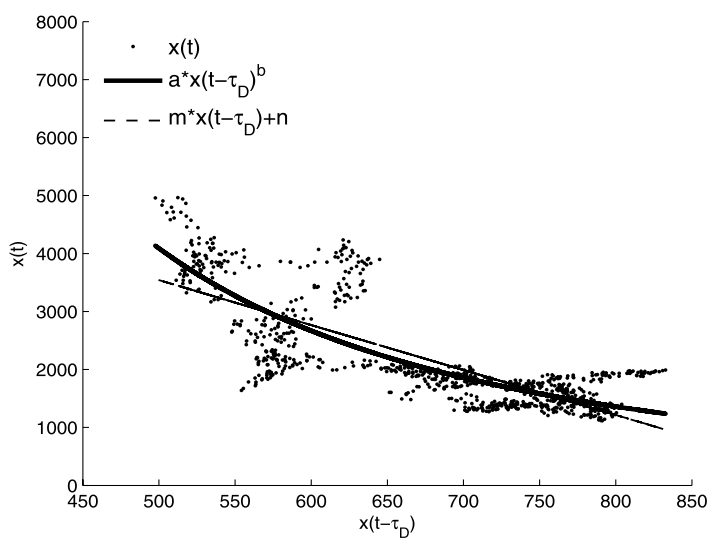

Partition 3

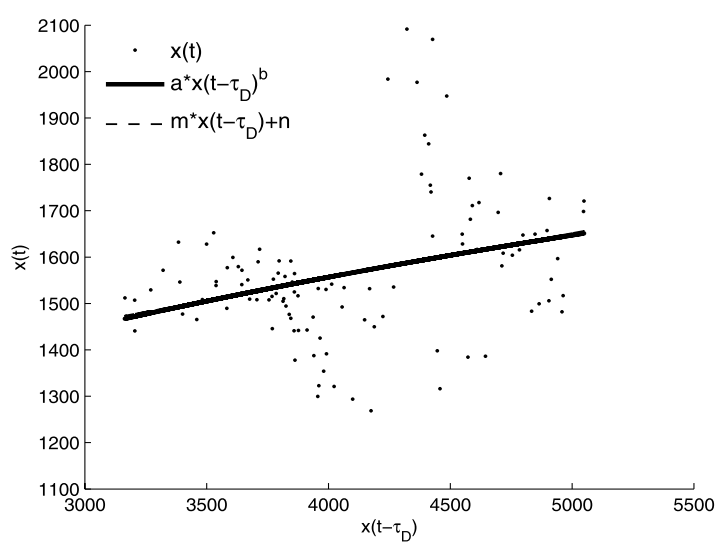

Partition 5

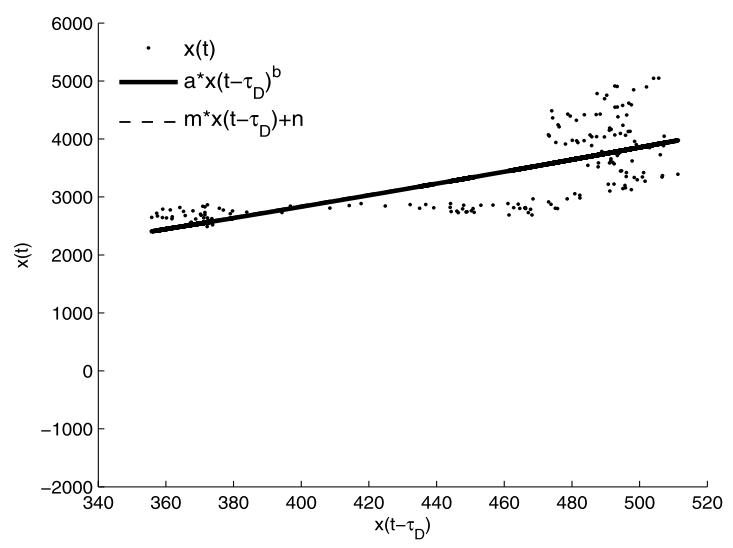

Partition 2

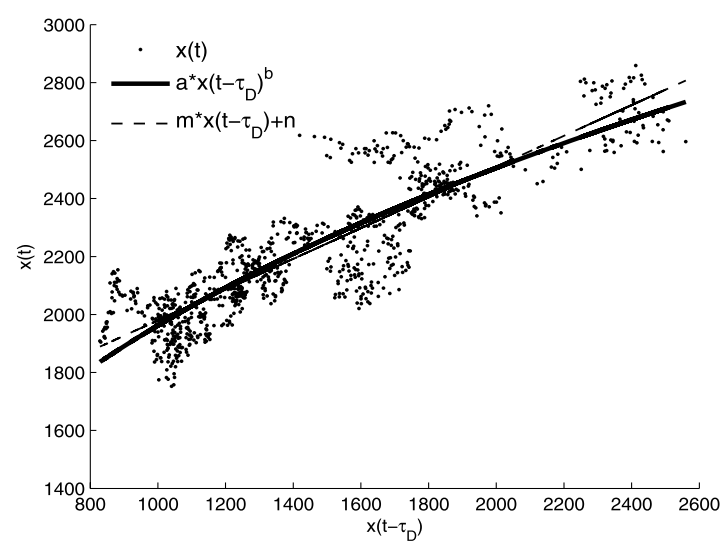

Partition 4

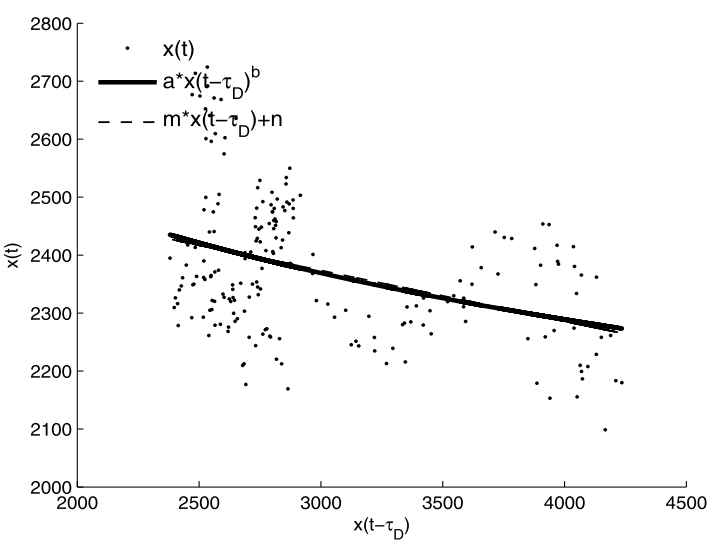

Partition 6

Fig. 12 The six partitions and corresponding power law and linear law trendlines for the NASDAQ index's $P P P$ 

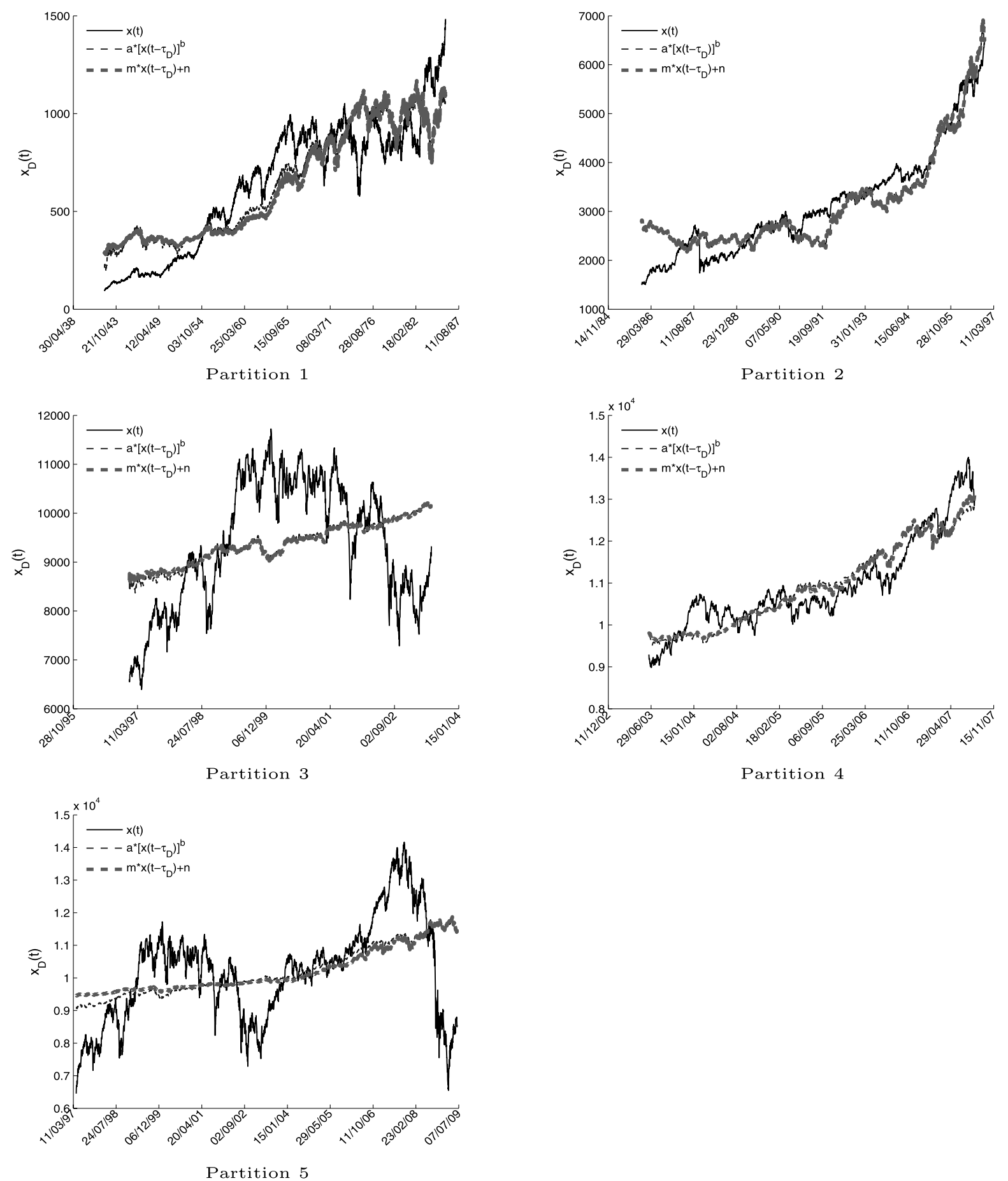

Fig. 13 Temporal data and corresponding power law and linear law mapping for the Dow Jones index 


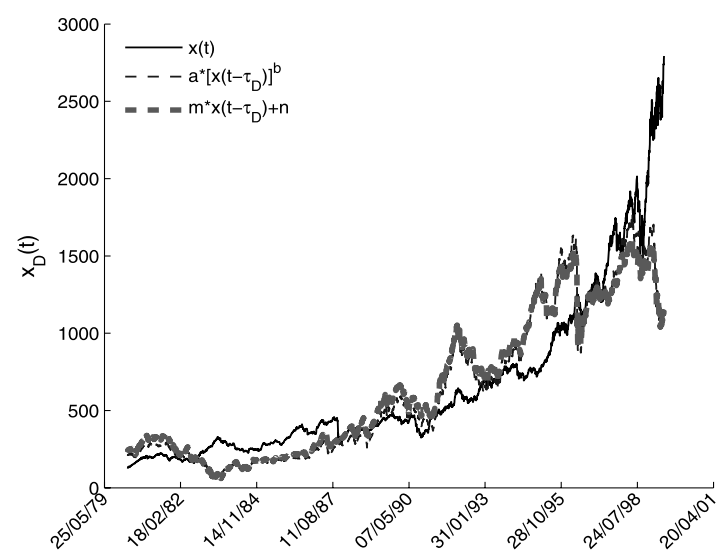

Partition 1

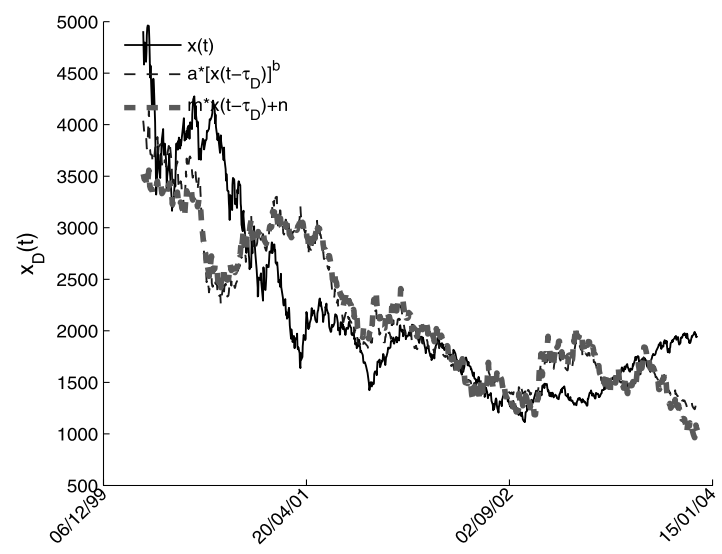

Partition 3

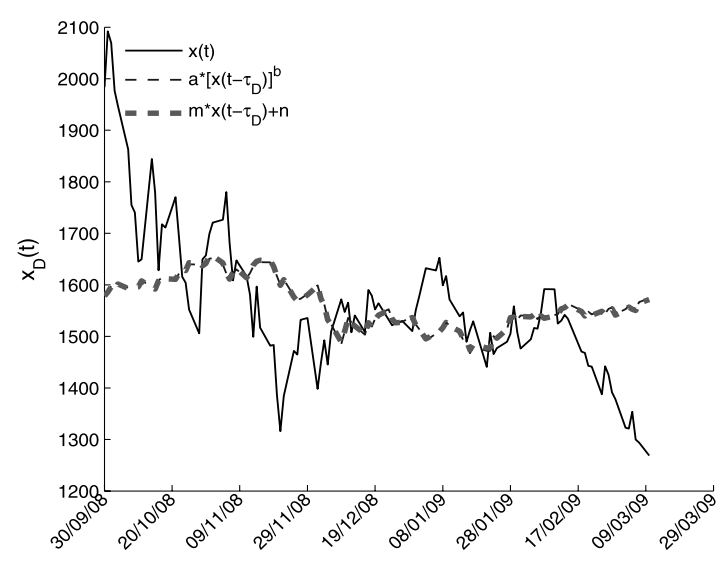

Partition 5

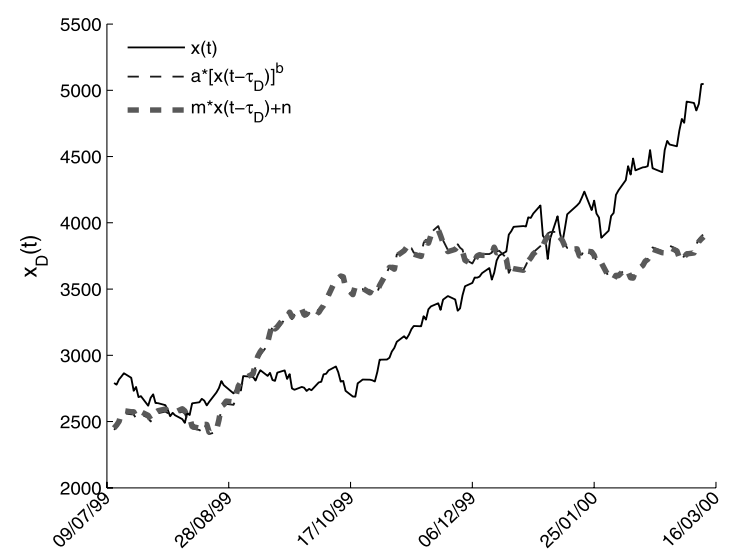

Partition 2

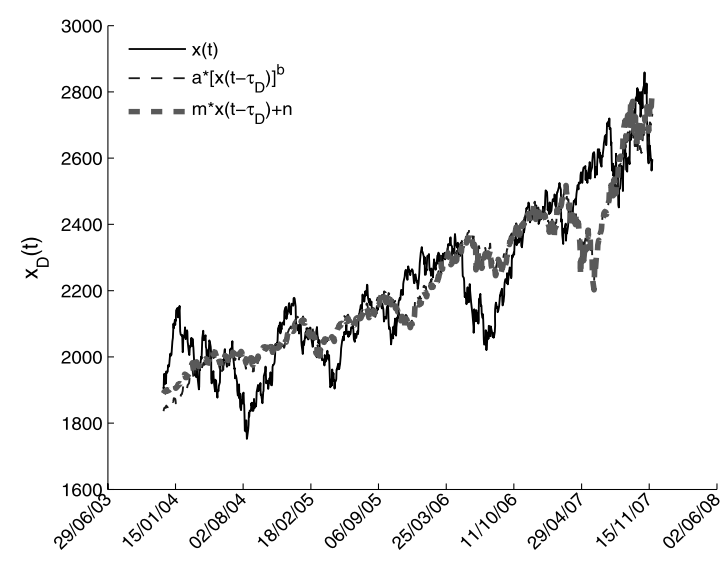

Partition 4

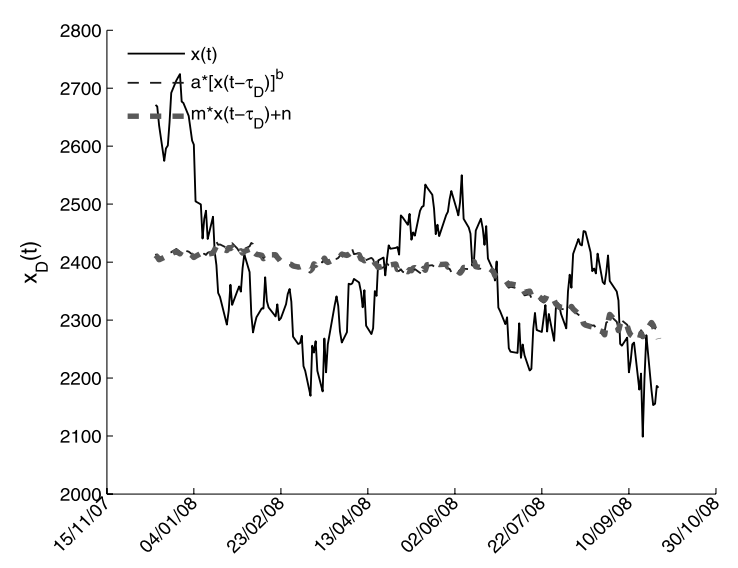

Partition 6

Fig. 14 Temporal data and corresponding power law and linear law mapping for the NASDAQ index 


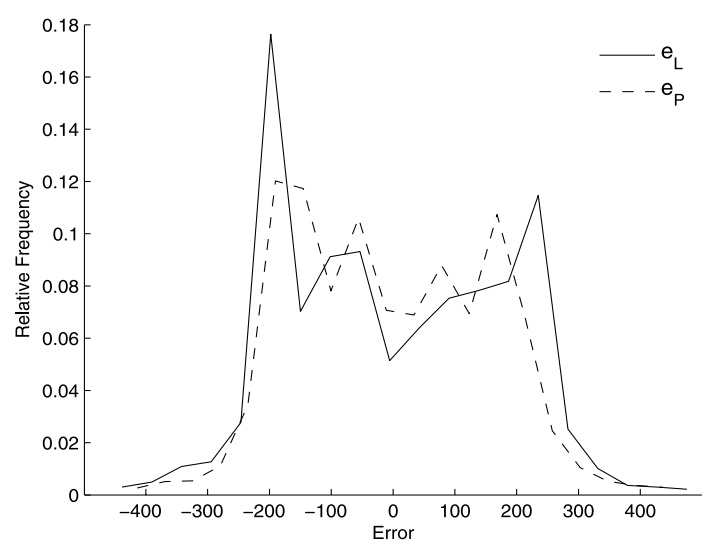

Partition 1

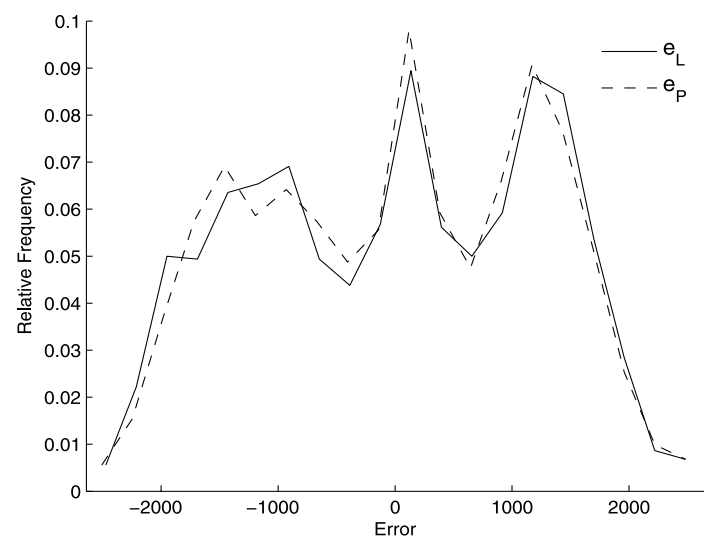

Partition 3

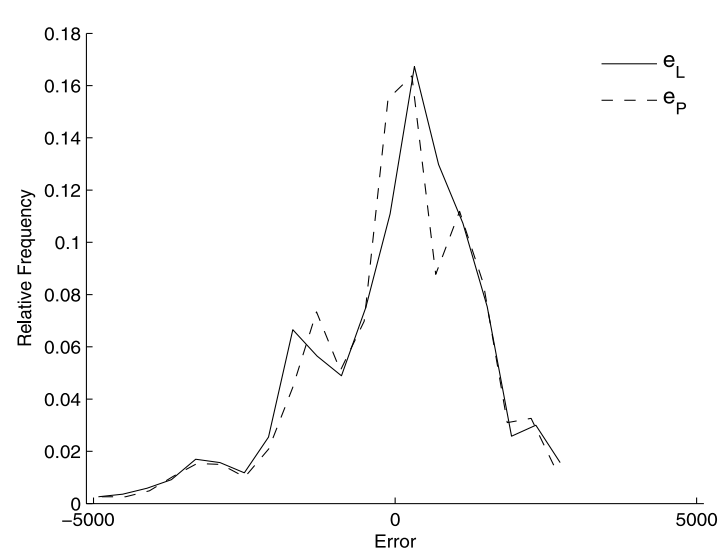

Partition 5

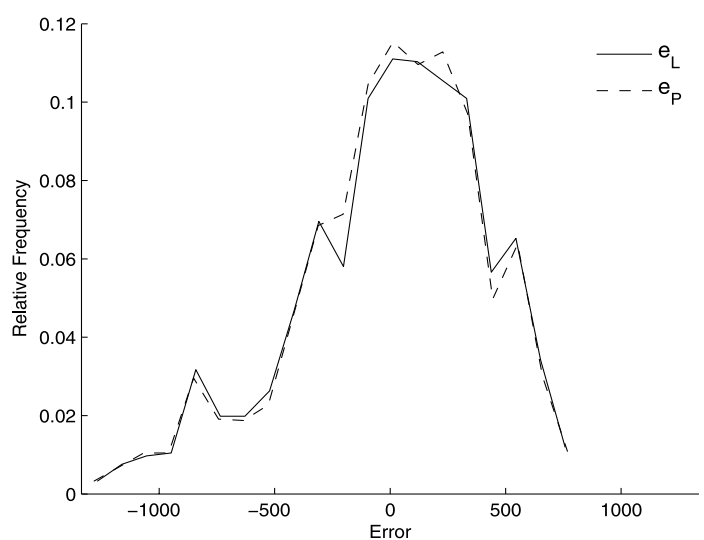

Partition 2

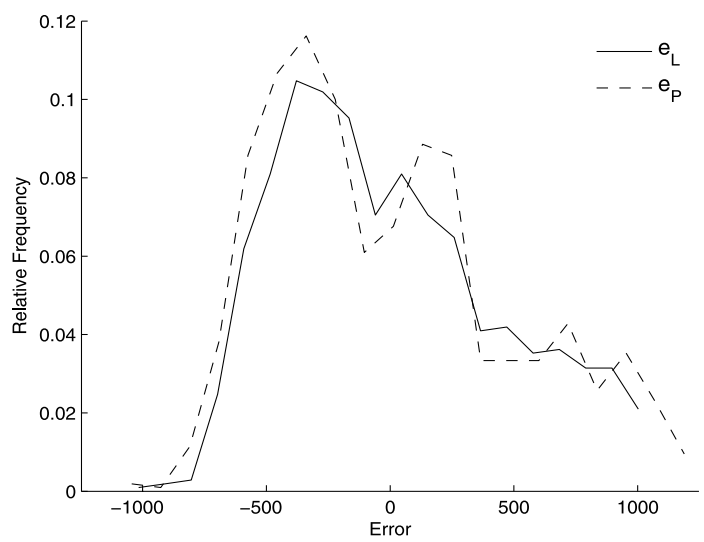

Partition 4

Fig. 15 Error histograms for the Dow Jones index: $e_{P}(t)=x(t)-a[x(t-\tau)]^{b}$ and $e_{L}(t)=x(t)-m x(t-\tau)-n$ 


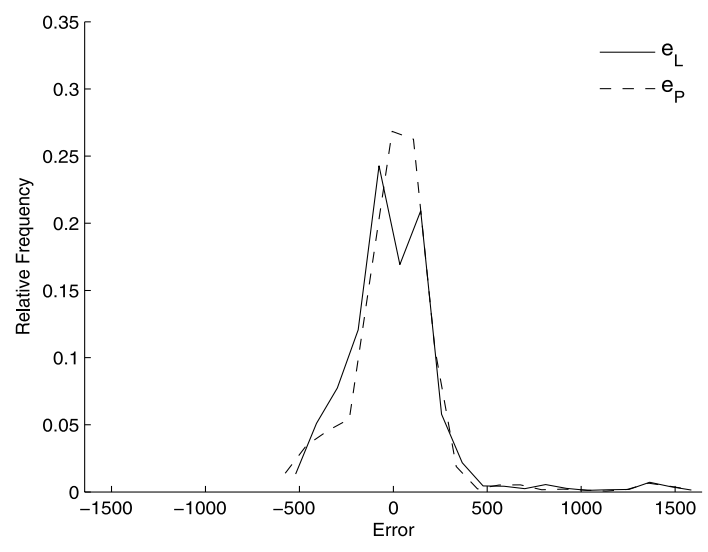

Partition 1

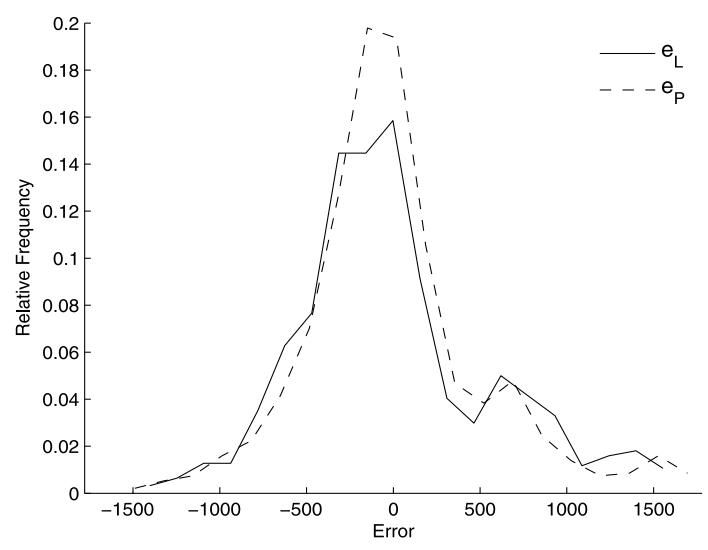

Partition 3

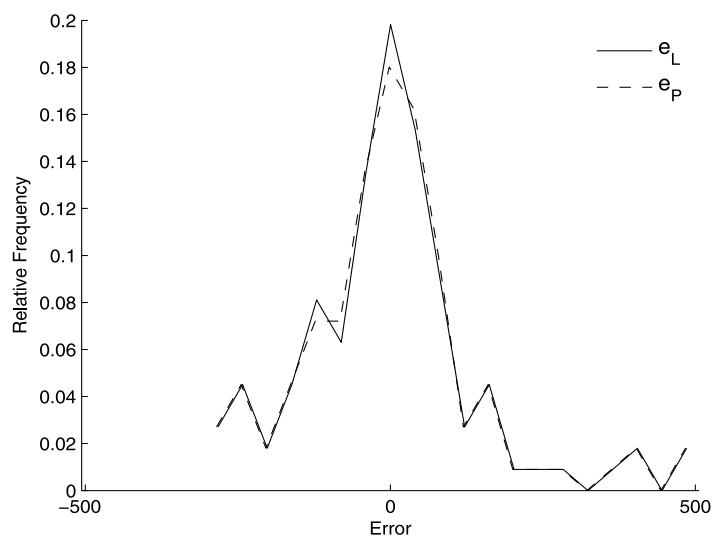

Partition 5

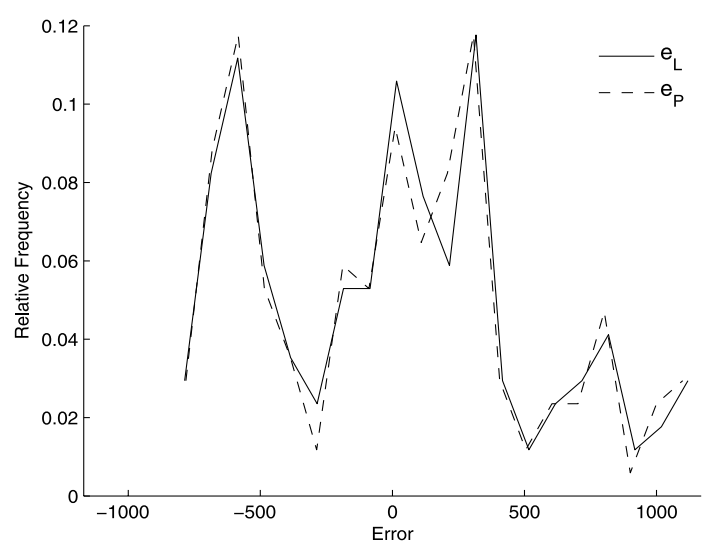

Partition 2

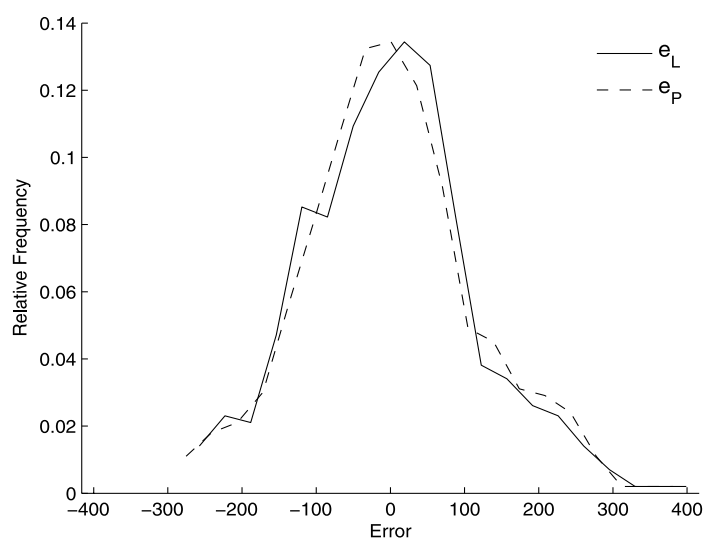

Partition 4

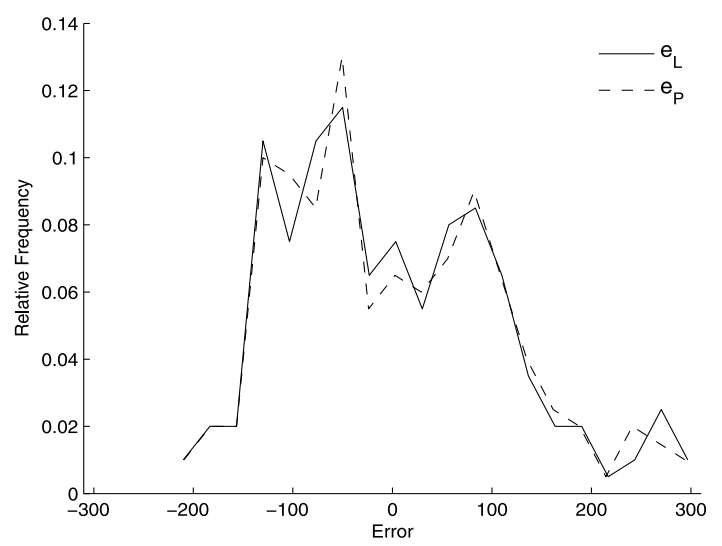

Partition 6

Fig. 16 Error histograms for the NASDAQ index: $e_{P}(t)=x(t)-a[x(t-\tau)]^{b}$ and $e_{L}(t)=x(t)-m x(t-\tau)-n$ 
Table 4 Parameters $\{m, n\}$ of the linear law trendline for the $P P P$ partitions, for the NASDAQ and the Dow Jones indexes

\begin{tabular}{llllll}
\hline Partition & \multicolumn{2}{l}{ Dow Jones } & & \multicolumn{2}{c}{ NASDAQ } \\
\cline { 2 - 3 } \cline { 5 - 6 } & $m$ & $n$ & & $m$ \\
\hline 1 & 0.8878 & 235.13 & & 3.6635 & -152.48 \\
2 & 2.3964 & 389.03 & & 9.8351 & -1080.1 \\
3 & 0.7549 & 7235.3 & -7.7537 & 7417.8 \\
4 & 0.618 & 7400.4 & & 0.5304 & 1449.4 \\
5 & 0.2465 & 8985 & & 0.0969 & 1167.0 \\
6 & & & -0.0875 & 2636 \\
\hline
\end{tabular}

Table 5 Arithmetic mean and standard deviation $\{\mu, \sigma\}$ of the error histograms for the Dow Jones index: $e_{P}(t)=x(t)-$ $a[x(t-\tau)]^{b}$ and $e_{L}(t)=x(t)-m x(t-\tau)-n$

\begin{tabular}{lrrrrr}
\hline Partition & \multicolumn{2}{l}{ Power law, $e_{P}$} & \multicolumn{2}{l}{ Linear law, $e_{L}$} \\
\cline { 2 - 3 } \cline { 5 - 6 } & \multicolumn{1}{l}{$\sigma$} & & \multicolumn{2}{c}{$\sigma$} \\
\hline 1 & -6.2484 & 156.8542 & -0.0031 & 175.3228 \\
2 & -4.0265 & 407.8812 & -0.0087 & 412.9503 \\
3 & -1.1110 & 1197.4871 & -0.06688 & 1223.0052 \\
4 & -0.9997 & 489.4155 & -0.19709 & 445.3473 \\
5 & -0.9680 & 1347.4192 & 0.1813 & 1398.3492 \\
\hline
\end{tabular}

the NASDAQ index's $P P P$ chart we consider six partitions as shown in Fig. 12.

For each of these partitions, power law $x(t)=$ $a x(t-\tau)^{b}$ and linear $x(t)=m x(t-\tau)+n$ trendlines are calculated. Tables 3 and 4 depict the values of the trendlines parameters $\{a, b\}$ and $\{m, n\}$ for both indexes.

For all of the PPP partitions we superimpose (Figs. 11 and 12), on the temporal data, the corresponding values of the power law and the linear trendlines mappings over the original data. Moreover, the corresponding errors, $e_{P}(t)=x(t)-a[x(t-\tau)]^{b}$ and $e_{L}(t)=x(t)-m x(t-\tau)-n$, and histograms are obtained and, for the each error type, the corresponding values for the arithmetic mean and the standard deviation are calculated. Tables 5 and 6 show the values of the arithmetic mean and the standard deviation $\{\mu, \sigma\}$ of the errors $e_{P}$ and $e_{L}$, for the Dow Jones and NASDAQ indexes, respectively.

Figures 13 and 14 depict the $P P P$ partitions and the trendlines approximation for the Dow Jones and the NASDAQ indexes, respectively, for both types of trendline mappings. Figures 15 and 16 depict the rela-
Table 6 Arithmetic mean and standard deviation $\{\mu, \sigma\}$ of the error histograms for the NASDAQ index: $e_{P}(t)=x(t)-$ $a[x(t-\tau)]^{b}$ and $e_{L}(t)=x(t)-m x(t-\tau)-n$

\begin{tabular}{lrrrrr}
\hline Partition & \multicolumn{2}{l}{ Power law, $e_{P}$} & & \multicolumn{2}{c}{ Linear law, $e_{L}$} \\
\cline { 2 - 3 } & $\mu$ & $\sigma$ & & \multicolumn{1}{c}{$\sigma$} \\
\hline 1 & 17.5949 & 265.2153 & -0.0117 & 276.8396 \\
2 & 1.5065 & 506.9526 & -0.0164 & 509.4117 \\
3 & 3.6146 & 517.0247 & 0.0118 & 555.1289 \\
4 & 0.1030 & 115.6174 & & 0.0379 & 113.8387 \\
5 & 0.0052 & 143.5438 & 0.1396 & 143.4068 \\
6 & -0.0002 & 110.92 & -0.0202 & 110.9426 \\
\hline
\end{tabular}

tive error's charts for the Dow Jones and the NASDAQ indexes, respectively.

These charts demonstrate that we have relationships between distinct time periods. This observation is consistent with the fractional order long-range memory effect depicted by the Fourier transform. At the present time it is neither clear what is the required number of partition to characterize completely the $P P P$, nor the relations between the trendline parameters and the Fourier transform details. Moreover, further research towards having the "best" trendline is needed. Nevertheless, it is clear that long-term memory relations exist and that more research efforts are still needed.

\section{Conclusions}

The Dow Jones and the NASDAQ indices were studied using several techniques usually adopted in dynamical systems. The Fourier spectrum of the Dow Jones and NASDAQ indexes was approximated by trendlines. Based on the slope of the trendlines the fractional order behavior was evidenced. To provide simultaneous insight in time and frequency behavior of the signal, we study the spectrum signals using a sliding-window Gaussian Fourier transform.

For the $P P P$ reconstruction an alternative technique based on the time series was also adopted. The time lag was calculated for each index signal using the first minimum value of the autocorrelation. The tests suggest that the time lag obtained for the minimum autocorrelation values leads to patterns of relationship between several time partitions. 


\section{References}

1. Dow, C.: The History of the Dow Jones Averages. (n.d). Retrieved September 11, 2009 from http://www.cftech. com/BrainBank/FINANCE/

2. The NASDAQ stock market. (n.d). Retrieved September 11, 2009 from http://www.essortment.com/all/

3. Retrieved June 18, 2009, from http://finance.yahoo.com/ $\mathrm{q} / \mathrm{hp}$ ? $\mathrm{s}=\% 5 \mathrm{EDJI}$

4. Retrieved July 23, 2009, from http://finance.yahoo.com/ $\mathrm{q} / \mathrm{hp}$ ? $=\% 5$ EIXIC

5. Takens, F.: Detecting strange attractors in turbulence. Dyn. Syst. Turbul. 898, 366-381 (1981)

6. Packard, N., Crutchfield, J., Farmer, D., Shaw, R.: Geometry from a time series. Phys. Rev. Lett. 45, 712-716 (1981)

7. Abarbanel, H.D., Brown, R., Tsimring, L.Sh.: The analysis of observed chaotic data in physical systems. Rev. Mod. Phys. 65(4), 1331-1392 (1993)

8. Plerou, V., Gopikrishnan, P., Rosenow, B., Amaral, L.A.N., Stanley, H.E: Econophysics: Financial time series from a statistical physics point of view. Physica A 279, 443-456 (2000)

9. Tenreiro Machado, J.A.: A probabilistic interpretation of the fractional-order differentiation. J. Fractional Calc. Appl. Analysis 6(1), 73-80 (2003)

10. Feeny, B.F., Lin, G.: Fractional derivatives applied to phase-space reconstructions. Nonlinear Dyn. 38(1-4), 8599 (2004)

11. Lima, M.F.M., Tenreiro Machado, J.A., Crisóstomo, M.: Fractional dynamics in mechanical manipulation. In: Proceedings of the ASME 2007 International Design Engineering Technical Conferences \& Computers and Infor- mation in Engineering Conference IDETC/CIE, 6th International Conference on Multibody Systems, Nonlinear Dynamics and Control (MSNDC). Las Vegas, NV, USA (2007)

12. Gabaix, X., Gopikrishnan, P., Plerou, V., Stanley, E.: A unified econophysics explanation for the power-law exponents of stock market activity. Physica A 382, 81-88 (2007)

13. de Lima, M.F.M.: Análise dinâmica de vibrações em manipuladores robóticos. Phd thesis, Faculdade de Ciências e Tecnologia, Univ. Coimbra, Coimbra (2008)

14. Mendes, R.V., Oliveira, M.J.: A data-reconstructed fractional volatility model. Econ. Open-Access OpenAssessment E-J. 2(22) (2008). http://www.economicsejournal.org/economics/discussionpapers/2008-22

15. Lima, M.F.M., Tenreiro Machado, J.A., Crisóstomo, M.: Pseudo phase plane, delay and fractional dynamics. J. Eur. Syst. Autom. 42, 1037-1051 (2008)

16. Jiang, Z.-Q., Zhou, W.-X., Sornette, D., Woodard, R., Bastiaensen, K., Cauwels, P.: Bubble diagnosis and prediction of the 2005-2007 and 2008-2009 Chinese stock market bubbles (2009). http://www.citebase.org/ abstract?id=oai:arXiv.org:0909.1007

17. Vilela Mendes, R.: A fractional calculus interpretation of the fractional volatility model. Nonlinear Dyn. 55(4), 395399 (2009)

18. Nigmatullin, R.R.: Universal distribution function for the strongly-correlated fluctuations: General way for description of different random sequences. Commun. Nonlinear Sci. Numer. Simul. 15(3), 637-647 (2010) 